\title{
Using a single band GNSS receiver to improve relative positioning in autonomous cars
}

\author{
Erik Stenborg ${ }^{1,2}$ and Lars Hammarstrand ${ }^{1}$
}

\begin{abstract}
We show how the combination of a single band global navigation satellite systems (GNSS) receiver, standard automotive level inertial measurement unit (IMU), and wheel speed sensors, can be used for relative positioning with accuracy on a decimeter scale. It is realized without the need for expensive dual band receivers, base stations or long initialization times. This is implemented and evaluated in a natural driving environment against a reference systems and against two simple base line systems; one using only IMU and wheel speed sensors, the other also adding basic GNSS. The proposed solution provides substantially slower error growth than either of the two base line systems.
\end{abstract}

\section{INTRODUCTION}

For autonomous vehicles, self-localization is one of the fundamental problems that needs to be solved. That is, knowledge of where the vehicle is located relative to the road is crucial for the vehicle to drive autonomously. For safety reasons, the solution needs to be accurate and fault tolerant, and in order to reach the market it must also be cost efficient.

A common approach to the self-localization problem is to equip the vehicle with external viewing sensors, such as cameras, lidars and radars, and a detailed map including landmarks that are visible with these sensors, e.g., lane markings, traffic signs, general image corner descriptors, or simply a rasterized image of the reflectivity of the road [1], [2], [3]. To locate oneself in the detailed map, the current observations from the onboard sensors are matched with the position of the landmarks stored in the map.

To be robust against electrical sensor failures, adverse weather, or blockage, the landmark based localization is combined with other means of localization. This is especially important in situations where there are few landmarks, or when the environment is different from what is stored in the map. Two candidates that are available at low marginal cost are the Global Navigation Satellite System (GNSS) receiver, and odometry from vehicle internal sensors such as inertial measurement units (IMU) and wheel speed sensors (WSS). In these cases, the GNSS receiver provides independent measurements of the global position to bound the error. The odometry sensors deliver frequent information about the movement of the vehicle from which one can perform dead reckoning. This helps to simplify the landmark based localization and to handle extensive failures for a limited time (enough time to make a safe stop).

\footnotetext{
${ }^{1}$ Signals and Systems at Chalmers University of Technology

${ }^{2}$ Volvo Car Corporation
}

These sensors also have their weaknesses and anomalies which, if not handled properly, would degrade the positioning performance. For example, GNSS receivers are highly susceptible to bad reception conditions, for instance in tunnels, next to large buildings, or because of atmospheric effects. Furthermore, survey grade GNSS receivers are still expensive, in part due to higher manufacturing cost and low volumes, but licensing fees for patents related to the L2 band may also contribute. The odometry sensors, on the other hand, only provide relative positioning information, and noise and biases in the sensors limit the ability to dead reckon for extended periods of time.

In this paper we investigate how to cost efficiently use a GNSS receiver, IMU and WSS to increase the accuracy and robustness of the self-localization during periods of external sensor failures or limited availability of landmarks. In the literature there are several ways to combine GNSS with IMU and wheels speed sensors. The level of integration, ranges from loosly coupled, to tightly coupled, to ultra-tightly or deeply coupled. Generally, a tighter coupling gives better performance, but adds complexity to the solution. Also the GNSS receiver architecture and algorithms greatly influence the positioning accuracy as well as the cost. There is a range from simple single band receivers which only use the Coarse/Acquisition code to dual band receivers also able to track the carrier phases. The more advanced systems that use the phase measurement of the carrier wave normally require solving the integer ambiguity problem [4]. Two popular techniques that solve this ambiguity are "real-time kinematics" (RTK), which uses relative positioning to a base station, and precise point positioning (PPP) which does not rely on having nearby base stations, but on the other hand needs a long convergence time [5]. Both the dependence on nearby base stations and long convergence time can be problematic in an automotive setting.

There are examples of how to use the more accurate but ambiguous carrier phase measurements in cheaper one channel receivers to improve the results without the use of base stations or long convergence times. One early such example would be carrier phase smoothing [6]. Slightly more recent examples include [7], [8], [9]. These works focus on the GNSS part, and do not investigate how a fused approach performs.

We propose a solution using the carrier phase measurement from a single band receiver tightly integrated with a standard grade IMU and WSS. To achieve the required accuracy we use occasional landmark observation to estimate 


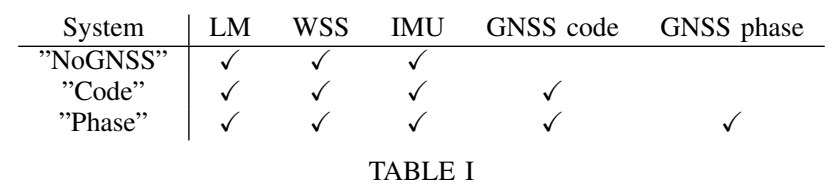

INFORMATION USAGE FOR THE THREE MODELS

the error states in both the GNSS and the odometry sensors. Additionally, instead of trying to estimate the absolute position of the vehicle, we focus on accurately estimating the odometry. As we are only concerned with the relative movement of the vehicle, we can use the more accurate phase measurement in a way that the integer ambiguity is no longer a problem. We evaluate the relative positioning performance of the proposed system against a reference system, and then compare it to two base line systems which only make use of basic GNSS signals (C/A code) or no GNSS signals. We make this comparison for a range of different dead reckoning times.

\section{Problem Formulation}

The aim of this paper is to analyze how much better a system that uses carrier phase observations from a single band GNSS receiver is for autonomous vehicle localization, when compared to systems not using it. We name the three systems that we compare according to their usage of GNSS measurements, see Table I, for easier reference in later sections.

We are interested in the relative positioning performance, so for each of these three systems, we estimate the vehicle pose and speed at times between the observation of landmarks, using the available data. During normal operations the time between landmark observations is short, but during, e.g., sensor failures, it can be much longer.

Let us introduce the discrete time index $k$ corresponding to the time $t_{k}$ that the $k$ :th measurement from any of the sensors was made. The parameters of interest are collected in the state vector

$$
\tilde{\mathbf{x}}_{k}=\left[\mathbf{p}_{k}^{T}, \boldsymbol{\theta}_{k}^{T}, v_{k}, \dot{\boldsymbol{\theta}}_{k}^{T}\right]^{T} .
$$

The sub-states, $\mathbf{p}_{k}=\left[e_{k}, n_{k}, u_{k}\right]^{T}, \boldsymbol{\theta}_{k}=\left[h_{k}, p_{k}\right]^{T}, v_{k}$, and $\dot{\boldsymbol{\theta}}_{k}=\left[\dot{h}_{k}, \dot{p}_{k}\right]^{T}$, represent position, orientation, speed, and turn rates, respectively, of the middle of the rear axle of the host vehicle. Although the pose of a rigid body is described by 3 coordinates for the position and 3 for the angles, we choose a more restricted model using only two angles, one for the heading and one for the pitch of the car. This is enough if we assume that the rear wheels of the car have no sideways motion, and thus the motion is independent of the roll angle.

The position is described using east, $e_{k}$, north, $n_{k}$, and up, $u_{k}$, coordinates in a Cartesian frame aligned with the curved system of longitudes and latitudes at some chosen linearization point, see Fig. 1. The orientation of the vehicle is expressed using the heading angle, $h_{k}$, with respect to the east-axis and the pitch angle, $p_{k}$, with respect to the tangent plane, see Fig 2.

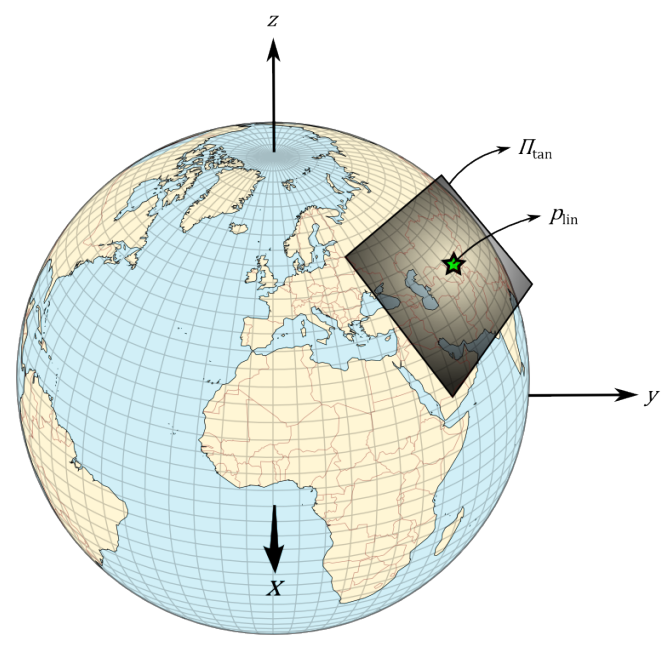

Fig. 1. The distance to the linearization point, $p_{\text {lin }}$, affects how well the north-, east-, and up-directions of the tangent plane, $\Pi_{\tan }$, align with the real directions on the Earth. If the height element in the state is 0, that position will be on $\Pi_{\tan }$, but the height over the Earth ellipsoid as reported by, e.g., a GNSS receiver will be greater than 0 everywhere but in $p_{\text {lin }}$. We use an arbitrary but static point in Göteborg city center for linearization.

Now, let us denote the set of all measurements received from all sensors up to and including time instance $k$ as $\mathbf{y}_{1: k}$. The aim is then to recursively calculate the posterior density $p\left(\tilde{\mathbf{x}}_{k} \mid \mathbf{y}_{1: k}\right)$ of the state $\tilde{\mathbf{x}}_{k}$ as defined in Eq. (1). From the posterior we can then calculate estimates of the pose of the host vehicle as well as relevant uncertainty measures.

\section{A. Information sources}

Each information source is presented in detail below starting with the GNSS receiver.

1) GNSS receiver: Every second the GNSS receiver outputs two signals for each visible satellite, $s$. We have the pseudo range measurement $\psi_{k}^{s}$ (based on the C/A code) and the carrier phase measurement $\phi_{k}^{s}$.

The pseudo range measurement is a coarse measurement of the Euclidean distance in meters between the receiver antenna and the satellite. A major source of uncertainties for this signal is the receiver time, which one usually treats as an unknown, and solve for together with x-, y-, z-position. Other errors affecting the accuracy of this measurement include, e.g., interference in the atmosphere and errors in the satellite clocks and orbits.

The carrier phase measurement, in contrast to its name, is a measure of the change in distance between the host and a satellite since the satellite was first observed. This is typically a more precise measurement than the pseudo range but in order to get an estimate of the actual range, one needs to find the initial range. This range is related to the unknown integer number of whole wavelengths of the carrier signal, between satellite and receiver at the first observation. The carrier phase measurement is affected by the same sources of error as the pseudo range measurements.

In addition to measuring pseudo range and carrier phase, the receiver also receives a navigation message that contains 


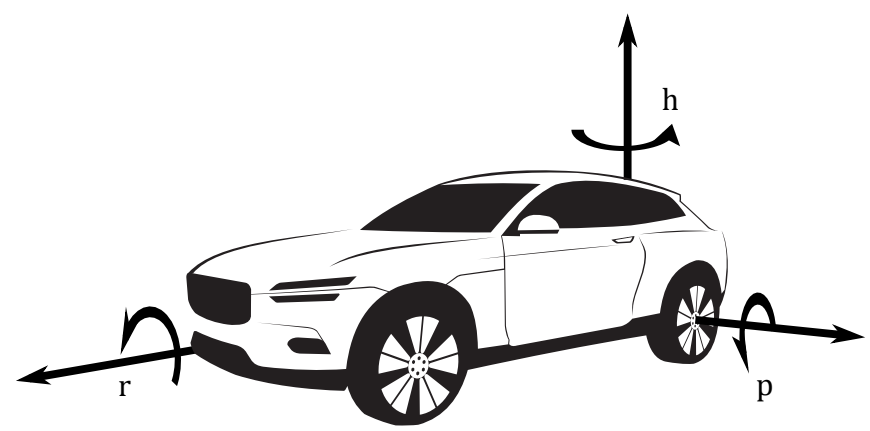

Fig. 2. The angles for describing the orientation of the car. The order of rotation is important, and is here chosen as first heading $(h)$, then pitch $(p)$ and last roll $(r)$. Heading is defined as counter-clockwise rotation from the east direction. Pitch is the rotation from the horizontal plane.

information about the system as a whole. Two important parts of this message that we depend on are the parameters describing the orbits of all satellites, and the parameters used to model some atmospheric delays.

2) Inertial measurement unit: The IMU contains a 3axis gyroscope measuring the angular velocities pitch rate, heading rate, and roll rate every $20 \mathrm{~ms}$. We denote these signals by $\omega_{k}^{\mathrm{p}}, \omega_{k}^{\mathrm{h}}$ and $\omega_{k}^{\mathrm{r}}$ for respective dimension, see Fig. 2 .

3) Wheel speed sensors: The vehicle is equipped with four wheel encoders, one in each wheel, measuring how many discrete fractions of a whole turn each wheel turns every $20 \mathrm{~ms}$. The corresponding angular velocity is scaled with a nominal wheel radius to approximate the speed of the wheel hub over ground. We denote the corresponding discrete time signals as $v_{k}^{\mathrm{fl}}, v_{k}^{\mathrm{fr}}, v_{k}^{\mathrm{rl}}, v_{k}^{\mathrm{rr}}$ for front left and right and rear left and right, respectively.

4) Landmark localization: In this paper, we use a synthetic position sensor that directly measures the position of the host vehicle. These simulated measurements are generated using a reference positioning system mounted on the host vehicle. That is, assuming that the reference positioning system gives us the position of the host vehicle at time $k$, the synthetic position measurement $\mathbf{p}_{k}^{l m}$ is generated as,

$$
\mathbf{p}_{k}^{\operatorname{lm}}=\mathbf{p}_{k}+\mathbf{r}_{k}^{\operatorname{lm}}
$$

where $\mathbf{r}_{k}^{\mathrm{lm}} \sim \mathcal{N}\left(0 ; I_{3 \times 3} R_{\mathrm{lm}}\right)$ is simulated measurement noise.

\section{MODELS}

In this section we describe the measurement models needed in the three systems as well as the process model for the state vector $\tilde{\mathbf{x}}_{k}$. In order to accurately describe the measurement models we need to add unknown and time varying parameters. These parameters are collected in a auxiliary state vector $\mathbf{b}_{k}$ and estimated jointly with $\tilde{\mathbf{x}}_{k}$.

\section{A. Measurement models}

1) Landmark localization: We assume that we have perfect knowledge regarding the positioning sensor, so we simply use the model that generated the observations (2) to also describe the measurements in our filter.
2) Wheel speed sensors: Because of the nominal radius assumed for the wheel speeds, the measured speeds must be multiplied by an unknown correction factor for each wheel, $r^{\mathrm{rr}}, r^{\mathrm{rl}}, r^{\mathrm{fr}}$, and $r^{\mathrm{fl}}$. However, as we know that the same type of tire is mounted on all four wheels, and we assume that the tread wear and air pressure is similar for all four wheels we approximate these four parameters with a very slowly varying correction factor $b_{k}^{\mathrm{ws}}$. We append this factor $b_{k}^{\mathrm{ws}}$ to the state vector and estimate it in the filter.

Further, the four wheel speeds, $v_{k}^{\mathrm{fl}}, v_{k}^{\mathrm{fr}}, v_{k}^{\mathrm{rl}}, v_{k}^{\mathrm{rr}}$, do not only give information about the speed of the car. They also give information about the heading rate of the vehicle. Introducing the lateral distance from the center of the car to the wheels, $l_{\mathrm{w}}$, and the wheel base, $l_{\mathrm{l}}$, the four individual wheel speeds are modeled as

$$
\begin{aligned}
& v_{k}^{\mathrm{fl}}=b_{k}^{\mathrm{ws}} \sqrt{\left(v_{k}-\dot{h}_{k} l_{\mathrm{w}}\right)^{2}+\left(\dot{h}_{k} l_{1}\right)^{2}}+r_{k}^{\mathrm{fl}} \\
& v_{k}^{\mathrm{fr}}=b_{k}^{\mathrm{ws}} \sqrt{\left(v_{k}+\dot{h}_{k} l_{\mathrm{w}}\right)^{2}+\left(\dot{h}_{k} l_{1}\right)^{2}}+r_{k}^{\mathrm{fr}} \\
& v_{k}^{\mathrm{rl}}=b_{k}^{\mathrm{ws}}\left(v_{k}-\dot{h}_{k} l_{\mathrm{w}}\right)+r_{k}^{\mathrm{rl}} \\
& v_{k}^{\mathrm{rr}}=b_{k}^{\mathrm{ws}}\left(v_{k}+\dot{h}_{k} l_{\mathrm{w}}\right)+r_{k}^{\mathrm{rr}},
\end{aligned}
$$

where

$$
\left[r_{k}^{\mathrm{fl}}, r_{k}^{\mathrm{fr}}, r_{k}^{\mathrm{rl}}, r_{k}^{\mathrm{rr}}\right]^{T} \sim \mathcal{N}\left(0 ; I_{4 \times 4} R_{v}\right) .
$$

is quantization noise from the wheel encoders.

3) Inertial measurement unit: We assume that the measurements from the IMU are corrupted by an additive, and slowly varying bias for each dimension, denoted $b_{k}^{\theta_{h}}$ and $b_{k}^{\theta_{p}}$, respectively. We further assume that pitch and roll angles are small, which gives us the following measurement equation

$$
\begin{aligned}
& \omega_{k}^{\mathrm{h}}=\dot{h}_{k}+b_{k}^{\mathrm{h}}+r_{k}^{\mathrm{h}} \\
& \omega_{k}^{\mathrm{p}}=\dot{p}_{k}+b_{k}^{\mathrm{p}}+r_{k}^{\mathrm{p}}
\end{aligned}
$$

where $\left[r_{k}^{\mathrm{h}}, r_{k}^{\mathrm{p}}\right]^{T} \sim \mathcal{N}\left(0 ; I_{2 \times 2} R_{\theta}\right)$ models measurement noise. The bias parameters, $b_{k}^{\mathrm{h}}$ and $b_{k}^{\mathrm{p}}$, are appended to the state vector.

4) GNSS receiver: For satellite $s$, the GNSS receiver produces two independent measurements of the Euclidean distance, $\rho_{k}^{s}$, between the receiver and the satellite, codebased pseudo range $\psi_{k}^{s}$ (used in the "Code" system) and carrier phase $\phi_{k}^{s}$ (used in the "Phase" system). In this section we present models for both of these and further explain how the carrier phase is used in the "Phase" system.

First let's define the distance $\rho_{k}^{s}$, which relates the satellite position, $x_{k}^{s}, y_{k}^{s}$, and $z_{k}^{s}$ known from the navigation message, to the receiver position, $x_{k}^{r}, y_{k}^{r}$, and $z_{k}^{r}$ as

$$
\rho_{k}^{s}=\sqrt{\left(x_{k}^{s}-x_{k}^{r}\right)^{2}+\left(y_{k}^{s}-y_{k}^{r}\right)^{2}+\left(z_{k}^{s}-z_{k}^{r}\right)^{2}} .
$$

These $x-, y-$, and $z$-coordinates are in an Earth centered and Earth fixed frame. The host position, $e_{k}, n_{k}$, and $u_{k}$ from (1) is aligned with the tangent plane in Fig. 1, which is related to the Earth centered frame through a linear transformation. The transform from the position as defined in the state vector to the GNSS receiver antenna position, also includes addition of the vector from the mid point between 
the wheels to the position of the GNSS antenna in the vehicle coordinate frame.

Using (10), the pseudo range measurements, $\psi_{k}^{s}$ used in the "Code" system, can be modeled as

$$
\psi_{k}^{s}=\rho_{k}^{s}+I_{k}^{s}+T_{k}^{s}+\Delta t_{k}^{s}+\Delta t_{k}^{r}+r_{k}^{\psi, s},
$$

where $I_{k}^{s}$ and $T_{k}^{s}$ are signal delays caused by the ionosphere and troposphere, respectively, $\Delta t_{k}^{r}$ is the receiver clock bias and $\Delta t_{k}^{s}$ represents satellite specific errors, such as satellite clock bias and orbit error. Note that, for convenience, all time delays and clock biases are implicitly multiplied by the speed of light in vacuum so that they are expressed in meters instead of seconds. The noise processes $r_{k}^{\psi, s} \sim \mathcal{N}\left(0 ; R_{\psi}\right)$ models measurement noise in the receiver.

The ionospheric delay, $I_{k}^{s}$, in (11) can be roughly calculated using Klobuchar's model [10] and parameters received in the navigation message. The remaining error is ignored, and contribute to an error in the calculated position. The tropospheric delay to each satellite, $T_{k}^{s}$, can be modeled as

$$
T_{k}^{s}=m\left(E_{k}^{s}\right) T_{k}^{\mathrm{z}}
$$

where $T_{k}^{z}$ is the zenith tropospheric delay, $E_{k}^{s}$ is the elevation angle of a certain satellite, and $m(\cdot)$ is a mapping function,

$$
m(E)=\frac{1.001}{\sqrt{0.002001+\sin ^{2}(E)}},
$$

accounting for the longer path through the troposphere when a satellite is not in zenith [11]. This leaves the following unknown parameters in (11) to be estimated by the filter, $T_{k}^{z}$, for the tropospheric delay, the satellite specific errors $\Delta t_{k}^{s}$ and the receiver clock drift $\Delta t_{k}^{r}$.

The "Phase" system uses the more accurate but ambiguous carrier phase measurements, $\phi_{k}^{s}$, to estimate $\tilde{\mathbf{x}}_{k}$. In order to express things in length units, we model the product of carrier phase measurements, $\phi_{k}^{s}$, and the nominal carrier wave length in vacuum, $\lambda$, as

$$
\lambda \phi_{k}^{s}=\rho_{k}^{s}-I_{k}^{s}+T_{k}^{s}+\rho_{0}^{s}+\Delta t_{k}^{s}+\Delta t_{k}^{\mathrm{r}}+r_{k}^{\phi, s},
$$

where $\rho_{0}^{s}$ is the unknown constant or phase ambiguity term expressed in length units, and $r_{k}^{\phi, s}$ is a noise term.

As we are mainly interested in odometry, it is enough to measure the distance that the host vehicle has traveled between two consecutive satellite observations, and by doing so, not having to handle the unknown phase ambiguity. So, we instead consider the difference between two consecutive phase measurements

$$
\begin{aligned}
\lambda \Delta \phi_{k}^{s}= & \lambda\left(\phi_{k}^{s}-\phi_{k-1}^{s}\right) \\
= & \left(\rho_{k}^{s}-\rho_{k-1}^{s}\right)-\left(I_{k}^{s}-I_{k-1}^{s}\right)+\left(T_{k}^{s}-T_{k-1}^{s}\right)+ \\
& \left(\Delta t_{k}^{s}-\Delta t_{k-1}^{s}\right)+\left(\Delta t_{k}^{r}-\Delta t_{k-1}^{r}\right)+\left(r_{k}^{\phi^{s}}-r_{k-1}^{\phi^{s}}\right) \\
\approx & \left(\rho_{k}^{s}-\rho_{k-1}^{s}\right)-\Delta I_{k}^{s}+\Delta T_{k}^{s}+\Delta \nabla t_{k}^{r}+\sqrt{2} r_{k}^{\Delta \phi^{s}} .
\end{aligned}
$$

Here we make the approximation that the satellite specific errors change slowly on the time scale we are interested in. The $\rho_{0}$ term disappears before the approximation, since it's the same irrespective of $k$ by definition. The $\Delta I_{k}$ and $\Delta T_{k}$ that we keep, are small and could be ignored, but since we have approximations that are easy to compute, and they have been shown to improve results in a time differenced solution [9], we keep them. In this way we trade some precision and having to remember $\rho_{k}$ and $\Delta t_{k}^{\mathrm{r}}$ over time, for a much smaller number of parameters in the state.

We augment the state with $\Delta \mathrm{p}$ and $\Delta \boldsymbol{\theta}$, which describe the position and orientation change since the last GNSS update. Then it is possible to calculate all $\rho_{k-1}^{s}$ using (10) at the next GNSS measurement. The motion model for $\Delta p$ is identical to the one for $\mathbf{p}$, with the only exception that it is reset to 0 every time a GNSS measurement is made.

\section{B. Augmented state vector and process model}

In addition to $\tilde{\mathbf{x}}_{k}$ as defined in (1), we append the auxiliary states, $\mathbf{b}_{k}$, used to describe the measurements models, to for the augmented state vectors,

$$
\mathbf{x}_{k}=\left[\tilde{\mathbf{x}}_{k}^{T}, \mathbf{b}_{k}^{T}\right]^{T} .
$$

As the auxiliary state vector depend on the observations that the system makes use of, we in fact get three different $\mathbf{x}_{k}$ depending on which $\mathbf{b}_{k}$ we use. We define these system dependent vectors,

$$
\begin{aligned}
\mathbf{b}_{k}^{\text {NoGnss }} & =\left[b_{k}^{\mathrm{ws}}, b_{k}^{\mathrm{h}}, b_{k}^{\mathrm{p}}\right]^{T} \\
\mathbf{b}_{k}^{\text {Code }} & =\left[\mathbf{b}_{k}^{\text {NoGnss }}, \Delta t_{k}^{\mathrm{r}}, \Delta^{2} t_{k}^{\mathrm{r}}, \Delta^{3} t_{k}^{\mathrm{r}}, T_{k}^{\mathrm{z}}, \Delta t_{k}^{1}, \ldots, \Delta t_{k}^{S}\right]^{T} \\
\mathbf{b}_{k}^{\text {Phase }} & =\left[\mathbf{b}_{k}^{\text {NoGnss }}, \Delta t_{k}^{\mathrm{r}}, \Delta^{2} t_{k}^{\mathrm{r}}, \Delta^{3} t_{k}^{\mathrm{r}}, \Delta \mathbf{p}_{k}^{T}, \Delta \boldsymbol{\theta}_{k}^{T}\right]^{T}
\end{aligned}
$$

where $\Delta^{2} t^{r}$ and $\Delta^{3} t^{r}$ are the first and second order time derivative of $\Delta t^{r}$.

1) Vehicle process model: If we make an assumption of no significant body side slip, we can define a motion model which integrates the angle rates, $\dot{\boldsymbol{\theta}}$, and forward speed, $v$, over some time to predict a new pose. We use a constant velocity and turn rate model for the vehicle motion. The continuous time derivative of the state

$$
\begin{aligned}
\dot{\tilde{\mathbf{x}}} & =\tilde{\mathbf{f}}(\tilde{\mathbf{x}})+\tilde{\mathbf{q}} \\
\tilde{\mathbf{f}}(\mathbf{x}) & =\left[v \cos h \cos p, v \sin h \cos p, v \sin p, \dot{h}, \dot{p}_{p}, \mathbf{0}_{1 \times 3}\right]^{T}
\end{aligned}
$$

defines the vehicle motion model in continuous time. The process noise vector, $\tilde{\mathbf{q}}$, is approximated as Gaussian, and is only non-zero for elements corresponding to zero elements in $\tilde{\mathbf{f}}(\mathbf{x})$. The remaining elements in $\tilde{\mathbf{q}}$ are zero.

We use the Euler approximation to find the the discrete time motion model

$$
\mathbf{x}_{k+1}=\mathbf{x}_{k}+T \tilde{\mathbf{f}}\left(\mathbf{x}_{k}\right)+\mathbf{q}_{k} .
$$

where $T$ is the sampling time and $\mathbf{q}_{k}$ is the discretized noise term.

2) Auxiliary state process model: The three parameters $\Delta t_{k}^{\mathrm{r}}, \Delta^{2} t_{k}^{\mathrm{r}}$, and $\Delta^{3} t_{k}^{\mathrm{r}}$ from the $\mathbf{b}_{k}$ vectors, describe the receiver time delay as a constant acceleration model. All other elements in the auxiliary state vectors are modeled as independent random walks with Gaussian noise. 


\section{IMPLEMENTATION AND EVALUATION}

Due to nonlinearities, both in the measurement model and in the process model, there is no closed-form solution to the posterior density. However, as the prediction times are short and the measurement noise relatively small, Gaussian approximations of the posteriors yield sufficient accuracy. Consequently, we have chosen to use a cubature Kalman filter [12] to calculate the first two moments of the posterior density.

Further, to handle occasional cycle slips in the phase measurements from the GNSS receiver we discard highly unlikely measurements from satellites. Carrier phase measurements that are more than $3 \sigma$ from the predicted measurement of the filter are simply not considered, with the measurements that are smaller being assumed to have a Gaussian noise distribution.

After implementing our proposed method "Phase", and the two base line methods "Code" and "NoGNSS", we evaluate the three methods on a data set. The evaluation is done by calculating the error to a highly accurate reference system for each method, and then comparing this error between the three methods.

\section{A. Scenario}

An inertial navigation system comprising an RTK GNSS receiver (Trimble BD982) tightly coupled to a high end IMU in a product branded as Applanix LV 220, serves as the reference. The reference system uses post processing to produce a smoothed solution of pose and velocity. It does so using the measurements from the whole data set and nearby reference stations from the SWEPOS network in a batch smoother. The resulting position estimate has an estimated standard deviation below $5 \mathrm{~cm}$, except when driving in a longer tunnel, and can serve as ground truth in this comparison.

The GNSS measurements used in the positioning filter are taken from the same reference receiver, but only raw measurements from L1 band are used. This provides data similar to what a simple one channel receiver would produce. However, the antenna that was coupled to the reference system is most likely more sensitive and also more resistant to multi path errors as compared to what a simpler antenna would be.

For the evaluation, landmarks are not measured using real sensors, but instead simulated using the ground truth data. The simulated landmarks give rise to measurements of the position of the car at even intervals.

The driven route, see Fig. 3, consists mainly of arterial roads with two lanes in each direction. It has one longer tunnel and a number of overpasses, but no urban areas with tall buildings around. The reason for this choice is that it represents the type of environment where first autonomous vehicles are expected to operate. We remove the data from the tunnel and a few longer parts where the car was totally stationary from the comparison, because they corrupt the comparison. In the tunnel the three methods are known to be identical and the large error there would drown the remaining part. The stationary parts are not representative of normal usage and may lead to overly optimistic results, if included.

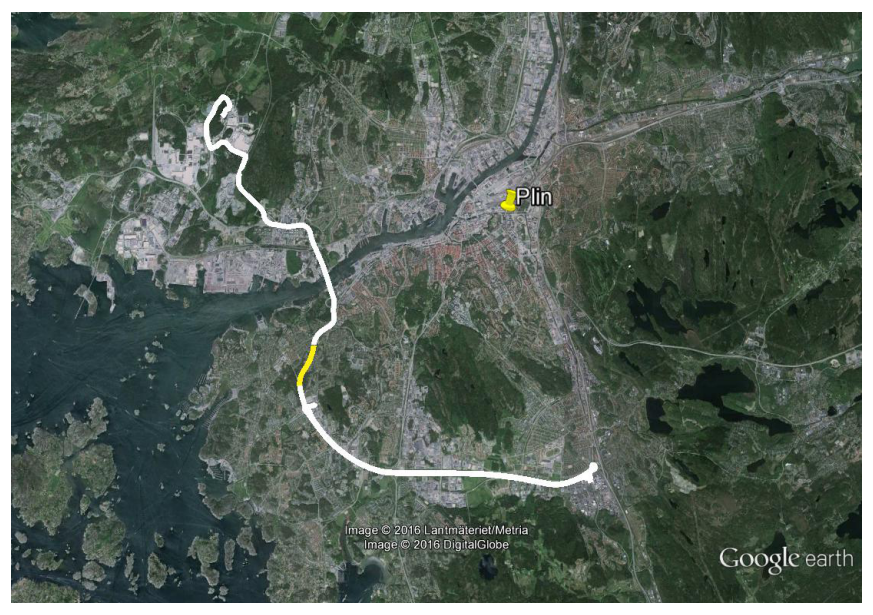

Fig. 3. Orthographic photo with the driven route overlaid. The white part of the route is from open sky conditions, with the exception of a number of overpasses, and the yellow part is inside a tunnel. The linearization point is marked with a pin.

\section{B. Results}

Since we have a good reference of the true position, we compare the three models we have implemented, "NoGNSS", "Code", and "Phase", to the ground truth. The output from our implementations is a sequence of estimates of the mean and covariance of the state vector.

The error in lateral position is one of the most important measures to get correct estimates for. If this error grows larger than a couple of decimeters, the result could be that the car crosses into an adjacent lane. For the plots we present here, we have projected the estimation error to this lateral position dimension, and are thus mainly comparing this measure and its variance between implementations and for various landmark frequency.

As we can see in Fig. 4, the error for the "NoGNSS" solution grows without bounds and quicker than linearly with the temporal length of the dead reckoning. As expected for the "Code" method, we get similar performance for short times, but the error is bounded by the precision of the GNSS measurements regardless of how long interval we have between the landmarks. This bound, however useful it is for normal navigation, is too large for autonomous driving. The "Phase" method on the other hand has error growth that is slow enough to be useful for the intended purpose up to about $10 \mathrm{~s}$ interval.

The RMSE is a rather crude performance measure, since we require a very low number of extreme errors for safety reasons. Although we haven't performed enough tests to get a reliable error distribution, we can get an indication by looking at the error histogram of the data we have, as in Fig. 5.

To judge the quality of the variance estimate, we tally the number of samples where the error is larger than a $\sigma$-level 


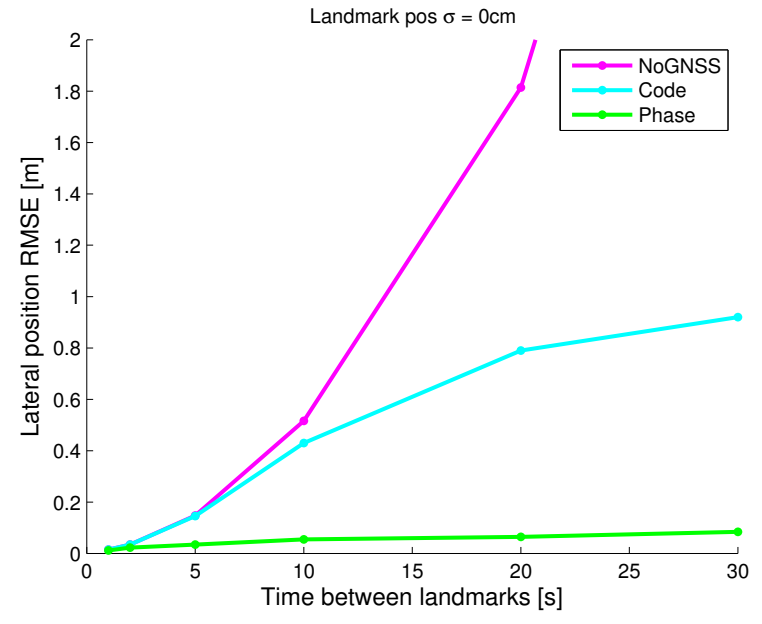

Fig. 4. Lateral root mean square error for three methods using different sets of measurement when compared to the RTK reference.
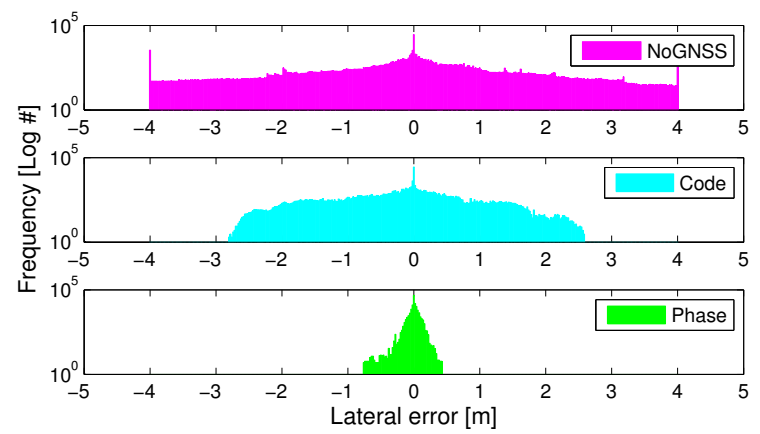

Fig. 5. Histogram of lateral error using logarithmic y-axis. Perfect landmark measurements every 20:th second. The outermost bars corresponds to all errors larger than $4 \mathrm{~m}$.

as estimated by the filter. Fig. 6 shows an example where the error exceeds the 3- $\sigma$ level for a number of samples. The amount of samples where error is large in this sense, is shown as a percentage of the total number of samples in the middle column in Table II.

\begin{tabular}{r|r|r}
$\mathrm{n}$ & amount large error & normal dist (for ref.) \\
\hline 1 & $12.73 \%$ & $31.73 \%$ \\
2 & $2.37 \%$ & $4.55 \%$ \\
3 & $0.55 \%$ & $0.27 \%$ \\
4 & $0.07 \%$ & $0.01 \%$ \\
5 & $0.00 \%$ & $0.00 \%$
\end{tabular}

TABLE II

AMOUNT OF DATA OUTSIDE THE ESTIMATED N- $\sigma$ LEVEL. THE NOMINAL VALUES FOR A NORMAL DISTRIBUTION ALSO INCLUDED FOR REFERENCE IN THE RIGHT COLUMN.

\section{CONCLUSiOnS}

In terms of autonomous driving, we already knew that regular GNSS receivers are not of much use besides providing a rough initialization for other localization methods. More tests with more production like hardware, and in more

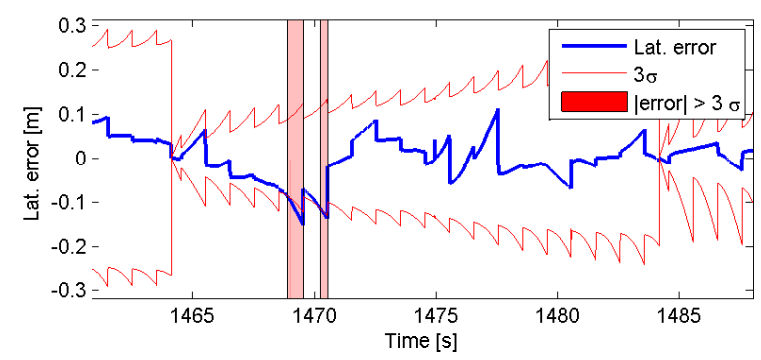

Fig. 6. Time plot of error, sigma level and region where error is larger than the sigma level.

environments are needed, but now we have an indication that by instead using a receiver capable of outputting carrier phase measurements, it is possible to make much better use of the GNSS receiver for relative positioning. The error growth is low enough for it to be useful on the time scales of 5-15 seconds that are needed to perform emergency stops.

\section{REFERENCES}

[1] J. Ziegler, P. Bender, M. Schreiber, H. Lategahn, T. Strauss, C. Stiller, T. Dang, U. Franke, N. Appenrodt, C. Keller, E. Kaus, R. Herrtwich, C. Rabe, D. Pfeiffer, F. Lindner, F. Stein, F. Erbs, M. Enzweiler, C. Knoppel, J. Hipp, M. Haueis, M. Trepte, C. Brenk, A. Tamke, M. Ghanaat, M. Braun, A. Joos, H. Fritz, H. Mock, M. Hein, and E. Zeeb, "Making bertha drive - an autonomous journey on a historic route," Intelligent Transportation Systems Magazine, IEEE, vol. 6, no. 2, pp. 8-20, Summer 2014.

[2] M. Lundgren, E. Stenborg, L. Svensson, and L. Hammarstrand, "Vehicle self-localization using off-the-shelf sensors and a detailed map," in IEEE Intelligent Vehicles Symposium, Proceedings, 2014, pp. $522-528$.

[3] J. Levinson and S. Thrun, "Robust vehicle localization in urban environments using probabilistic maps," in International Conference on Robotics and Automation, 2010.

[4] D. Kim and R. B. Langley, "Gps ambiguity resolution and validation: methodologies, trends and issues," in Proceedings of the 7th GNSS Workshop-International Symposium on GPS/GNSS, Seoul, Korea, vol. 30, no. 2.12, 2000.

[5] E. Kaplan and C. Hegarty, Understanding GPS: Principles and Applications, Second Edition, ser. Artech House mobile communications series. Artech House, 2005.

[6] R. Hatch, "The synergism of gps code and carrier measurements," in International geodetic symposium on satellite doppler positioning, vol. 1,1983 , pp. 1213-1231.

[7] T. J. Ford and J. Hamilton, "A new positioning filter: Phase smoothing in the position domain," Navigation, vol. 50, no. 2, pp. 65-78, 2003.

[8] S. Martin and D. M. Bevly, "Comparison of gps-based autonomous vehicle following using global and relative positioning," International Journal of Vehicle Autonomous Systems, vol. 10, no. 3, pp. 229-255, 2012.

[9] J. Traugott, G. DellOmo, A. Vyssotski, D. Odijk, and G. Sachs, "A time-relative approach for precise positioning with a miniaturized 11 gps logger," in Proceedings of the 21 st International Technical Meeting of the Satellite Division of the Institute of Navigation ION GNSS, 2008, pp. 1883-1894.

[10] J. Klobuchar et al., "Ionospheric time-delay algorithm for singlefrequency gps users," Aerospace and Electronic Systems, IEEE Transactions on, no. 3, pp. 325-331, 1987.

[11] H. Black and A. Eisner, "Correcting satellite doppler data for tropospheric effects," Journal of Geophysical Research: Atmospheres (1984-2012), vol. 89, no. D2, pp. 2616-2626, 1984.

[12] I. Arasaratnam and S. Haykin, "Cubature kalman filters," Automatic Control, IEEE Transactions on, vol. 54, no. 6, pp. 1254-1269, 2009. 